\title{
Harnessing the Power of Biologic Agents on the Oral Microbiota: A Way to Promote Oral and Systemic Health?
}

\author{
Claudio Passariello ${ }^{1}$, Dario Di Nardo ${ }^{2}$, Marco Seracchiani ${ }^{3}$, Shilpa Bhandi ${ }^{4}$, Luca Testarelli ${ }^{5}$
}

The Journal of Contemporary Dental Practice (2020): 10.5005/jp-journals-10024-2949

During the long history of their evolution, higher organisms, including mammals, have learnt to take great advantage from living in close contact with selected populations of microbes. 'By living in close contact, animals and microbes underwent a progressive and mutual co-evolutive process that is believed to be a major driving force in the development of adaptive immunity of vertebrates. ${ }^{2}$

As a result of this co-evolutive process, humans and other animals are characterized by their own unique microbiomes, each consisting of many hundred species of viruses, bacteria, archaea, fungi, and protozoa, unevenly distributed to colonize the different accessible regions of the body. ${ }^{3}$ The human microbiome is believed to account for $1-3 \%$ of body weight and to comprise more than 100 trillion cells. ${ }^{4}$ The microbiota is involved in complex host-microbe and microbe-microbe interactions, thus modulating nutrient acquisition, adjusting immune system development and general homeostasis (via epigenetic modifications of host genes ${ }^{5}$ ), and playing the role of protective barrier to pathogens. ${ }^{4}$ When a microbiota undergoes qualitative and quantitative changes with regard to distribution in a site and metabolic activity, this condition is defined dysbiosis and is expected to be associated with local and/ or distant pathologic signs. ${ }^{6}$

Bacterial products released by a dysbiotic microbiota interact with homeostatic mechanisms of the human host and cooperate to the pathogenesis of major human diseases, including diabetes mellitus, inflammatory bowel syndrome, atherosclerosis, obesity, liver disease, and cancer. ${ }^{4,7}$

The oral microbiota is the second more abundant and one of the most diverse and unique microbial communities in the human body. ${ }^{8}$ Although many of the most relevant oral and dental diseases, including caries, periodontal and peri-implant diseases, have been long recognized as of microbial origin, only recently the application of culture independent molecular methods using $16 \mathrm{~S}$ rRNA gene comparative analyzes enabled us to understand that they are caused by dysbiosis rather than by the action of specific pathogens. $^{9-11}$

The effects of oral dysbiosis are not limited to oral tissues: periodontal diseases, for example, are among the most common human diseases and their associations with diabetes, cardiovascular disease, metabolic disease and obesity, rheumatoid arthritis, certain cancers, respiratory diseases, and cognitive disorders is now supported by increasing evidence. ${ }^{12}$

Recent molecular investigations showed that some potentially pathogenic oral bacteria (named pathobionts), as for example Porphyromonas gingivalis, Aggregatibacter actinomycetemcomitans, and Fusobacterium nucleatum, colonize in low numbers the oral cavity of healthy individuals, without alerting sentinel systems of mucosal defences. ${ }^{13,14}$ Conditions able to disrupt the eubiotic equilibrium promote the overgrowth of pathobionts, which suddenly become pathogens (with support from commensals
${ }^{1}$ Department of Public Health and Infectious Diseases, Sapienza University of Rome, Rome, Italy

2,3,5 Department of Oral and Maxillo-Facial Sciences, Sapienza University of Rome, Rome, Italy

${ }^{4}$ Department of Restorative Dental Sciences, Jazan University, Jazan, Kingdom of Saudi Arabia

Corresponding Author: Luca Testarelli, Department of Oral and Maxillo-Facial Sciences, Sapienza University of Rome, Rome, Italy, Phone: +39 3381504134, e-mail: luca.testarelli@uniroma1.it

How to cite this article: Passariello C, Di Nardo D, Seracchiani M, et al. Harnessing the Power of Biologic Agents on the Oral Microbiota: A Way to Promote Oral and Systemic Health? J Contemp Dent Pract 2020;21(10):1073-1074.

Source of support: Nil

Conflict of interest: None

becoming accessory pathogens) and promote local and systemic inflammation by Treg and Th17 responses that, in turn, can induce diseases as atherosclerosis, Alzheimer's disease, inflammatory bowel disease, macular degeneration, and chronic kidney disease. ${ }^{13}$

Moreover, chronic microaspiration of oral microorganisms during oral dysbiosis was demonstrated to act as an important risk factor for the onset of a variety of lung diseases including asthma, COPD, pulmonary fibrosis, and pneumonia. ${ }^{15,16}$

The abovementioned considerations highlight the importance of maintaining/restoring a balanced oral microbiota for oral and general health. As for the more extensively investigated gut microbiota, many are the factors that influence the composition and equilibrium of the oral microbiota during life. ${ }^{17}$ Among these factors, pro-eubiotic biologic agents (including probiotic bacteria, bacteriophages, and predatory bacteria) deserve particular attention for future research and application. ${ }^{17}$

Probiotics are defined as nonpathogenic live microorganisms which, when administered in adequate amounts, confer benefits to the host's health. ${ }^{18}$ Some probiotic microorganisms are members of the normal oral microbiota, and their concentrations are reduced in the presence of dysbiosis. ${ }^{19,20}$ Probiotics have been investigated as means in the treatment/prevention of oral diseases. They were shown possibly able to contrast halitosis, ${ }^{21}$ oral fungal infections, ${ }^{22}$ to reduce oral counts of cariogenic bacteria, and clinical markers of periodontal inflammation. ${ }^{23,24}$ However, the level of evidence in this field is still insufficient, mainly due to the use of a wide range of probiotics, to non-standardized study designs and to the limited number of oral derived probiotic strains that could have more chances of prolonged oral permanence after administration. If we really intend to manage oral and systemic health with the help of probiotics, future studies should more precisely address the 
specific effects of single probiotics, adopting standardized study designs on larger populations and including an evaluation of the duration of clinical effects and oral colonization by each probiotic strain. Such evaluations should also be extended to some predatory bacterial species, since they were shown to be members of the oral microbiome, ${ }^{25}$ and their role as pro-eubiotic microorganisms was already demonstrated in the gut. ${ }^{26}$

\section{References}

1. Dethlefsen L, McFall-Ngai M, Relman DA. An ecological and evolutionary perspective on human-microbe mutualism and disease. Nature 2007;449(7164):811-818. DOI: 10.1038/nature06245.

2. Weaver CT, Hatton RD. Interplay between the TH17 and TReg cell lineages: a (co-) evolutionary perspective. Nat Rev Immunol 2009;9(12):883-889. DOI: 10.1038/nri2660.

3. Lloyd-Price J, Mahurkar A, Rahnavard G, et al. Strains, functions and dynamics in the expanded human microbiome project. Nature 2017;550(7674):61-66. DOI: 10.1038/nature23889.

4. Wang $B$, Yao M, Lv L, et al. The human microbiota in health and disease. Engineering 2017;3(1):71-82. DOI: 10.1016/J.ENG.2017.01.008.

5. Takahashi K, Sugi Y, Nakano K, et al. Epigenetic control of the host gene by commensal bacteria in large intestinal epithelial cells. J Biol Chem 2011;286(41):35755-35762. DOI: 10.1074/jbc.M111.271007.

6. Altveş S, Yildiz HK, Vural HC. Interaction of the microbiota with the human body in health and diseases. Biosci Microbiota Food Healt 2020;39(2):23-32. DOI: 10.12938/bmfh.19-023.

7. Blum HE. The human microbiome. Adv Med Sci 2017;62(2):414-420. DOI: 10.1016/j.advms.2017.04.005.

8. Li K, Bihan M, Yooseph S, et al. Analyses of the microbial diversity across the human microbiome. PLoS One 2012;7(6):e32118. DOI: 10.1371/journal.pone.0032118.

9. Marsh PD, Moter A, Devine DA. Dental plaque biofilms: communities, conflict and control. Periodontol 2000 2011;55(1):16-35. DOI: 10.1111/j.1600-0757.2009.00339.x.

10. Nyvad B, Takahashi N. Integrated hypothesis of dental caries and periodontal diseases. J Oral Microbiol 2020;12(1):1710953. DOI: 10.1080/20002297.2019.1710953.

11. Kröger A, Hülsmann C, Fickl S, et al. The severity of human periimplantitis lesions correlates with the level of submucosal microbial dysbiosis. J Clin Periodontol 2018;45(12):1498-1509. DOI: 10.1111/ jcpe.13023.

12. Genco RJ, Sanz M. Clinical and public health implications of periodontal and systemic diseases: an overview. Periodontol 2000 2020;83(1):7-13. DOI: 10.1111/prd.12344.

13. Meghil MM, Cutler CW. Oral microbes and mucosal dendritic cells, "Spark and Flame" of local and distant inflammatory diseases. Int J Mol Sci 2020;21(5):1643. DOI: 10.3390/ijms21051643.
14. Fine $\mathrm{DH}$, Schreiner $\mathrm{H}$, Velusamy SK. Aggregatibacter, a low abundance pathobiont that influences biogeography, microbial dysbiosis, and host defense capabilities in periodontitis: the history of a bug, and localization of disease. Pathogens 2020;9(3):179. DOI: 10.3390/ pathogens9030179.

15. Gaeckle NT, Pragman AA, Pendleton KM, et al. The oral-lung axis: the impact of oral health on lung health. Respir Care 2020;65(8):12111220. DOI: 10.4187/respcare.07332[Epub ahead of print].

16. O'Donnell LE, Smith K, Williams C, et al. Dentures are a reservoir for respiratory pathogens. J Prosthodont 2016;25(2):99-104. DOI: 10.1111/ jopr.12342.

17. Gagliardi A, Totino V, Cacciotti F, et al. Rebuilding the gut microbiota ecosystem. Int J Environ Res Public Health 2018;15(8):E1679. DOI: 10.3390/ijerph15081679.

18. Hill C, Guarner F, Reid G, et al. Expert consensus document. the international scientific association for probiotics and prebiotics consensus statement on the scope and appropriate use of the term probiotic. Nat Rev Gastroenterol Hepatol 2014;11(8):506-514. DOI: 10.1038/nrgastro.2014.66.

19. Koll-Klais $\mathrm{P}$, Mandar R, Leibur E, et al. Oral lactobacilli in chronic periodontitis and periodontal health: species composition and antimicrobial activity. Oral Microbiol Immunol 2005;20(6):354-361. DOI: 10.1111/j.1399-302X.2005.00239.x.

20. Wescombe PA, Hale JD, Heng NC, et al. Developing oral probiotics from Streptococcus salivarius. Future Microbiol 2012;7(12):1355-1371. DOI: 10.2217/fmb.12.113.

21. Burton JP, Chilcott CN, Moore CJ, et al. A preliminary study of the effect of probiotic Streptococcus salivarius K12 on oral malodour parameters. J Appl Microbiol 2006;100(4):754-764. DOI: 10.1111/j.13652672.2006.02837.x.

22. Song YG, Lee SH. Inhibitory effects of Lactobacillus rhamnosus and Lactobacillus casei on Candida biofilm of denture surface. Arch Oral Biol 2017;76:1-6. DOI: 10.1016/j.archoralbio.2016.12.014.

23. Passariello C, Gigola P, Testarelli L, et al. Evaluation of microbiota associated with herpesviruses in active sites of generalized aggressive periodontitis. Ann Stomatol (Roma) 2017;8(2):59-70. DOI: 10.11138/ads/2017.8.2.059.

24. Nguyen $\mathrm{T}$, Brody $\mathrm{H}$, Lin GH, et al. Probiotics, including nisin-based probiotics, improve clinical and microbial outcomes relevant to oral and systemic diseases. Periodontol 2000 2020;82(1):173-185. DOI: 10.1111/prd.12324.

25. Passariello C, Schippa S, Di Nardo D, et al. Molecular detection of the predatory bacterium Bdellovibrio bacteriovorus from dental biofilms. Dent Hypotheses 2019;10(4):103-107. DOI: 10.4103/denthyp. denthyp_95_19.

26. lebba V, Santangelo F, Totino V, et al. Higher prevalence and abundance of Bdellovibrio bacteriovorus in the human gut of healthy subjects. PloS One 2013;8(4):e61608. DOI: 10.1371/journal. pone. 0061608 